Revue d'études américaines. American Studies Journal

$2 \mid 2017$

(Hi)stories of American Women: Writings and Rewritings / Call and Answer: Dialoguing the American West in France

\title{
Antoine Traisnel, Hawthorne. Blasted Allegories
}

\section{Julien Nègre}

\section{OpenEdition}

\section{Journals}

Édition électronique

URL : https://journals.openedition.org/transatlantica/10521

DOI : 10.4000/transatlantica.10521

ISSN : 1765-2766

Éditeur

Association française d'Etudes Américaines (AFEA)

Référence électronique

Julien Nègre, "Antoine Traisnel, Hawthorne. Blasted Allegories », Transatlantica [En ligne], 2 | 2017, mis en ligne le 19 avril 2019, consulté le 01 février 2023. URL : http://journals.openedition.org/ transatlantica/10521 ; DOI : https://doi.org/10.4000/transatlantica.10521

Ce document a été généré automatiquement le 1 février 2023

\section{c) (7) $९$}

Creative Commons - Attribution - Pas d'Utilisation Commerciale - Pas de Modification 4.0 International - CC BY-NC-ND 4.0

https://creativecommons.org/licenses/by-nc-nd/4.0/ 


\section{Antoine Traisnel, Hawthorne. Blasted Allegories}

Julien Nègre

\section{RÉFÉRENCE}

Antoine Traisnel, Hawthorne. Blasted Allegories, Paris, Aux Forges de Vulcain, 2015, 504 pages, ISBN-10 : 2919176811, $20 €$

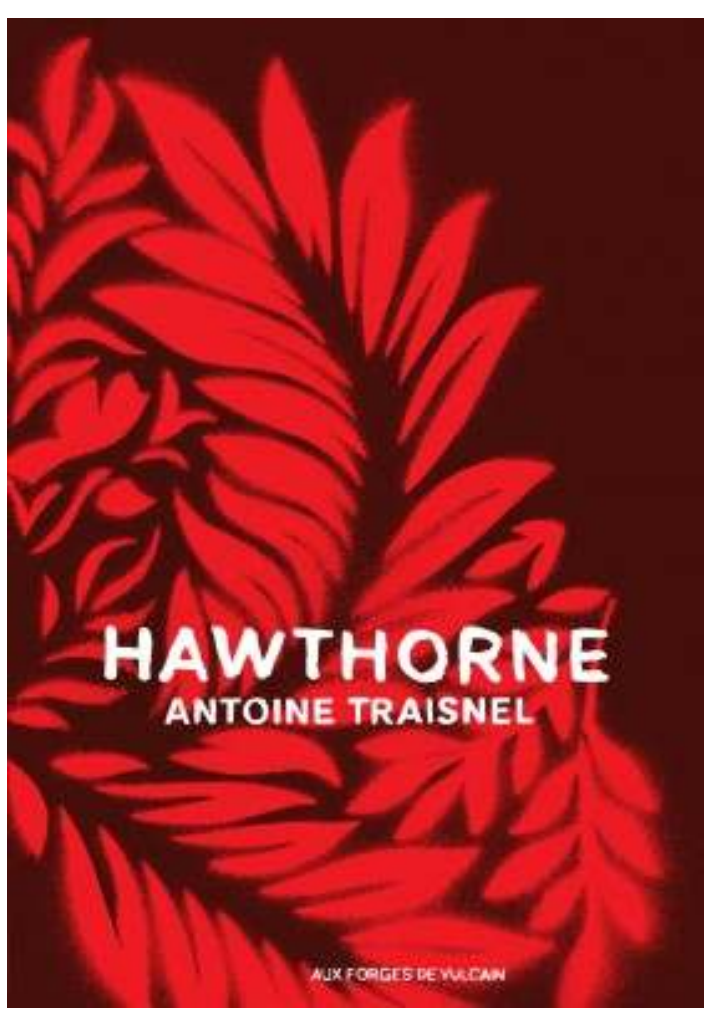

Couverture de l'ouvrage. Source : Aux Forges de Vulcain (http://www.auxforgesdevulcain.fr/ collections/essais/hawthorne/) 
Les flamboyantes efflorescences qui ornent la couverture du Hawthorne d'Antoine Traisnel semblent évoquer aussi bien le nom de la maison d'édition qui le publie (Aux Forges de Vulcain) que la lettre écarlate dont il est question dans le second (ou premier? nous y reviendrons) chapitre - et bien sûr tout au long de ce volume. Et comme la lettre écarlate elle-même, la couverture dissimule autant qu'elle révèle, et pointe du doigt autant qu'elle détourne l'attention. Un sous-titre accompagne en effet le titre de ce livre dense, exigeant et riche : Blasted Allegories, qui ne se donne à voir que sur la page de titre, à l'intérieur de l'objet. Un sous-titre (tiré d'une lettre écrite par Hawthorne en 1854) qu'Antoine Traisnel a judicieusement choisi de conserver en anglais, et dont il explore la polysémie et la richesse dans la préface de l'ouvrage (15-20) en soulignant que l'expression peut vouloir dire à la fois " maudites allégories ", «allégories flétries" (donc mortifères), et "allégories éclatées » (voire explosives). L'allégorie est donc le cachalot de cette étude des quatre romances (et bien plus) de Hawthorne. Tel le cétacé dans le roman de Melville (autre écrivain cher à l'auteur), elle occupe le cœur monumental et problématique de cette lecture, tout en disparaissant parfois sous la surface pendant de longs passages, pour mieux brécher à nouveau au détour d'un paragraphe de conclusion ou d'un point d'étape.

La préface («L'effacement de l'auteur ») s'ouvre ainsi sur le troublant daguerréotype de Hawthorne pris par John Adams Whipple, non pas dans sa version restaurée, mais dans celle conservée à la Bibliothèque du Congrès sur une plaque métallique abîmée et rayée. Ce portrait trouble et déconcertant de l'auteur, dont le regard disparaît dans les ombres irrégulières de la surface sensible, sert de point de départ pour dessiner avec clarté ce qui sera la problématique centrale de l'ouvrage: la place de l'allégorie comprise comme «régime de signification qui échoue à représenter une totalité cohérente » (42) ou plutôt qui, à l'inverse du symbole, « ne représente plus » (43), dans les textes de Hawthorne. 


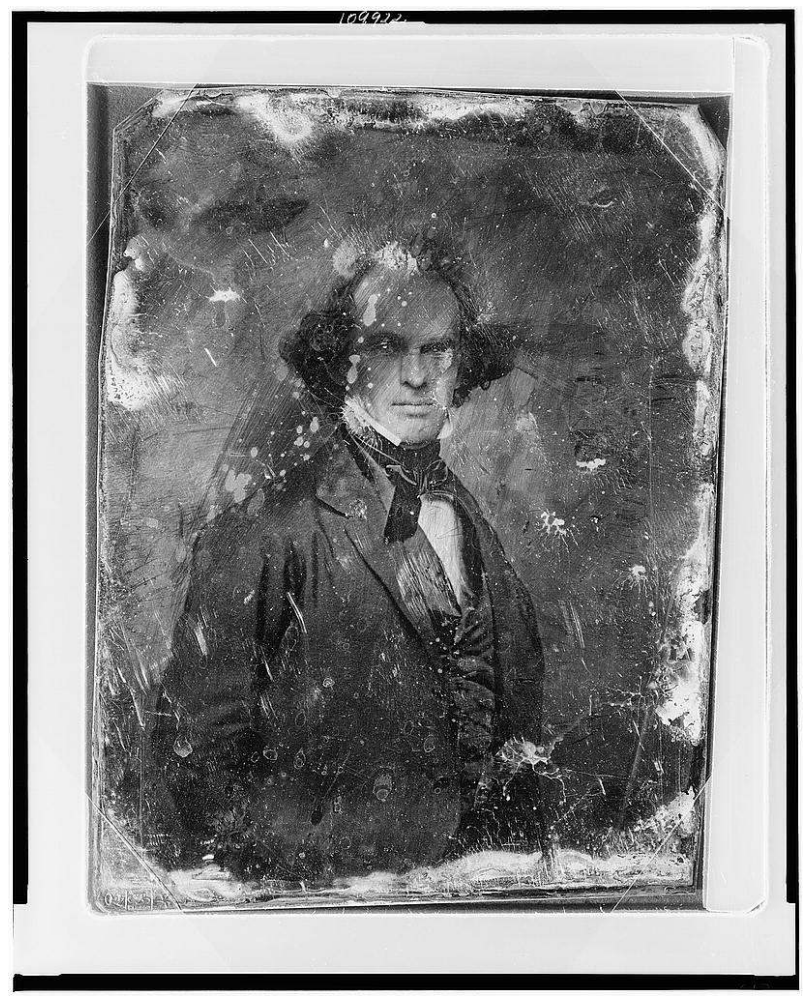

Portrait de Nathaniel Hawthorne, 1848. Source : Wikimedia Commons (https:// commons.wikimedia.org/wiki/File :Nathaniel_Hawthorne_daguerreotype.jpg).

3 À l'image de ces marques qui ornent à la fois le portait et certains personnages de l'auteur (telle Georgiana dans "The Birthmark»), l'allégorie serait cette "cicatrice » qui signe «l'impossible coïncidence de l'écriture avec son sujet, la persistance d'un interstice » (15) et par laquelle Hawthorne se donne à voir tout en s'effaçant sans cesse. Notant combien les écrits de Hawthorne, en mettant en scène son propre effacement par le biais de figures auctoriales fictives (M. de l'Aubépine, M. du Miroir), « anticipent, mettent en scène et remettent en question leur réception critique ", Antoine Traisnel se propose ainsi d'accomplir un mouvement circulaire qui englobe à la fois les textes eux-mêmes et leur réception critique, faisant pour cela le choix, paradoxal et prometteur méthodologiquement, de «partir de la critique pour ensuite revenir aux romances afin de soumettre cette critique à l'examen de la fiction » (45).

L'ouvrage suit pour cela un plan à la fois complexe et aisément lisible. Une première partie de 80 pages environ («L'allégorie ou l'écriture de l'après-coup ») est consacrée à la question de l'allégorie elle-même, et notamment celle de l'allégorie « critique ». Cette longue ouverture est l'occasion d'un dialogue fécond entre des perspectives critiques différentes, au cours duquel les textes de Hawthorne eux-mêmes ont tendance à passer temporairement à l'arrière-plan. Ils sont de nouveau placés au centre, cependant, dans les quatre chapitres suivants, dont chacun est consacré à une romance différente :

5 Sens Litéral - S. L.

6 Sens Typologique - The House of the Seven Gables et le sens de l'Histoire

7 Sens Moral - Blithedale démoralisée

8 Sens Anagogique - The Marble Faun ou les ruines de l'art 
9 Le chapitre consacré à The Scarlet Letter est ainsi le second de l'ouvrage mais marque le début des lectures des romances à proprement parler. Comme A. Traisnel l'explique à la fin du premier chapitre (111 et 123-125), le plan retenu ici suit les quatre niveaux d'interprétation de l'allégorie dans l'allégorèse médiévale. Ces quatre points d'entrée permettent à Antoine Traisnel d'étudier les articulations et les tensions que l'allégorie hawthornienne entretient avec la lettre (lettre typographique du A ou du petit a, mais aussi sens littéral qui, s'il est désigné comme sens premier, n'est pas toujours le premier sens), l'H/histoire (interrogée à travers la question de la lecture typologique, notamment puritaine), la morale (comme sens supplémentaire qui découle du texte, mais plus souvent le précède et donc conditionne sa lecture), et enfin avec la question de la fin (celle des temps, du texte et du corpus) et des limites du sens.

Dès l'ouverture de la première partie, Antoine Traisnel suit le programme annoncé en préface selon lequel l'objet de cette étude est à la fois le texte de Hawthorne et sa réception critique. L'« entame » de cette section s'interroge ainsi sur le point de départ le plus pertinent du corpus, et fait le choix d'aller à rebours de la lecture critique la plus habituelle qui verrait dans The Marble Faun non seulement la fin mais aussi le déclin du travail d'écriture, œuvre de "crise » et «nadir " de l'œuvre (50). En partant donc de The Marble Faun plutôt que des contes et nouvelles, Antoine Traisnel propose de lire l'allégorie comme " un régime d'écriture et non comme une simple figure de discours ", qui permet « d'accuser sans les résoudre les tensions critiques qui façonnent l'écriture de Hawthorne » (51-52). Tout en empruntant à l'allégorie "classique » l'irréductible écart que celle-ci maintient entre le sens littéral et le sens figuré qu'elle désigne, Antoine Traisnel propose ainsi de lire le travail d'écriture de Hawthorne comme une « allégorie critique » qui « laisse entrevoir dans le même temps la trace et le sens qu'elle exprime (le signifiant et le signifié) sans pour autant les confondre » (53). La portée de cette allégorie critique est explorée dans cette première partie de l'ouvrage. Le propos est ici étayé par de nombreuses références critiques et théoriques qui sont richement mises en dialogues. L'allégorie critique de Traisnel fait ainsi se rejoindre la différance derridienne (la distance entre le texte vu et le texte lu de l'allégorie n'étant jamais un « vide ») et le pli deleuzien (le " défaut de certitude » [64] de l'allégorie entraînant la prolifération éminemment baroque des points de vue).

11 Mouvement vers un sens autre qui trahit sans cesse sa propre artificialité, l'allégorie montre ainsi sous la plume de Traisnel sa «temporalité " propre (101), à la fois problématique et féconde : "chez Hawthorne le littéral ne s'approche qu'à force de déconstruction patiente d'un sens allégorique qui constitue, sinon le sens premier, du moins le premier sens, celui qui se donnerait d'entrée de jeu. Le caractère à la fois secondaire (censé découler du sens littéral) et premier (accessible avant le sens littéral qu'il faut recouvrer) du sens allégorique en fait un sens foncièrement ambigu, pour ne pas dire différant. " (198). Cette ambiguïté, pour Antoine Traisnel, est précisément ce qui donne à l'allégorie son " potentiel critique ». Loin de chercher à résoudre ou aplanir les tensions et contradictions qui habitent l'écriture de Hawthorne et la façon dont nous comprenons aujourd'hui l'homme et surtout "l'Auteur » qu'il a été, la posture critique de Traisnel consiste ainsi davantage à les mettre au jour et à les lire comme des stratégies délibérées qui démultiplient les potentialités du texte. Ainsi, alors qu'il commente la façon dont Hawthorne apparie ses romances d'une "morale » plus ou moins bricolée et plus ou moins pertinente, Antoine Traisnel note : "Chez Hawthorne, c'est mon hypothèse, arraisonner l'histoire à une morale n'aurait pas pour but d'en 
déterminer la fin, au double sens de terme et de finalité, mais servirait au contraire de par le hiatus qui distingue les deux modes de discours, à travers le jeu de différences qui s'installe entre le récit de fiction et l'énoncé souverain de la morale - à relancer indéfiniment le processus interprétatif » (289).

Malgré sa grande technicité, le style est clair et agréable. Les lectures sont appuyées sur de (parfois très) longues citations des textes de Hawthorne en français, souvent assorties de la version originale en anglais pour les passages les plus cruciaux. Tout en prenant beaucoup de hauteur, l'auteur se penche en effet avec méticulosité sur les mots et la langue de Hawthorne. Cette attention se retrouve dans les sous-titres évocateurs des différentes sections, qui permettent à Antoine Traisnel de déployer tous les sens des termes auxquels il se confronte. La "lettre » de The Scarlet Letter est ainsi à la fois initiale, parasite, voyelle, capitale, capitulation, décapitation, nom propre. Les ruines de l'art dans The Marble Faun sont l'occasion de discuter «l'art de la fin » mais aussi «le jeu, ou l'art d'accommoder les restes ». Les passionnantes analyses sur l'émergence du kitsch comme réponse à la rupture moderne dans la pratique artistique au $\mathrm{XIX}^{\mathrm{e}}$ montrent ainsi comment les «fausses » ruines, avec le temps, finissent par devenir tout aussi vénérables et moussues que les originales : «c'est peut-être dans la puissance du faux, suggère la romance, que s'origine véritablement l'art, qui a perdu son caractère facétieux au fil du temps » (413).

Avec Derrida et Deleuze, Walter Benjamin et Paul de Man sont les grandes figures critiques qui guident la réflexion d'un bout à l'autre de l'ouvrage. Au fil des analyses et des (micro-)lectures, Antoine Traisnel discute aussi avec rigueur les lectures de Fredric Jameson, Sacvan Bercovitch, Michael Colacurcio ou encore Patricia Crain, pour n'en citer que quelques-uns. Ce faisant, l'auteur développe une lecture particulièrement féconde et nuancée. L'ouvrage d'Antoine Traisnel constitue en cela non seulement une étape marquante dans les études hawthorniennes françaises et mondiales, mais il s'adresse aussi à ceux qui s'intéressent aux études américaines dans leur ensemble, ainsi qu'à la théorie critique en général.

INDEX

Thèmes : Recensions

\section{AUTEURS}

JULIEN NÈGRE

ENS Lyon 